\title{
Effect of Different Cooking Method on Cooking Loss and Lipid Oxidation in Buffalo Meat
}

\author{
Phongchai Klinhom ${ }^{1, a^{*}}$, Jitra Klinhom ${ }^{2, b}$, and Sasithorn Methawiwat ${ }^{3, c}$ \\ ${ }^{1,3}$ Agricultural Science Program, Faculty of Science and Technology, Kamphaeng Phet Rajabhat \\ University, Kamphaeng Phet, 62000, Thailand \\ ${ }^{2}$ Department of Product Development Technology, Faculty of Agro-Industry, Chiangmai University, \\ Chiang Mai, 50100, Thailand \\ aaggie-phong@hotmail.com; bj.klinhom09@gmail.com, \\ 'me27sasi@gmail.com
}

Keywords: Cooking method; Cooking loss; Lipid oxidation; Buffalo meat

\begin{abstract}
The aim of this work was to study the influence of different cooking methods (grilling, roasting, boiling, steaming and frying) on cooking loss and lipid oxidation of buffalo meat (Semimembranosus). Cooking induces significantly cooking loss and lipid oxidation (TBARs index $)$ in meat product $(P<0.05)$. When the different cooking methods were examined, grilling method showed lesser extent of cooking loss and higher level of MDA concentration compared to steaming which was observed with higher cooking loss but lower level of MDA concentration $(P<0.05)$. There were no significant differences on cooking loss and MDA concentration among the rest of the cooking methods $(P>0.05)$. The cooking loss was found to be related to MDA level with $\mathrm{r}=-0.54(P<0.05)$. It has been suggested in this study that cooking loss could be possible response to MDA level in buffalo meat product.
\end{abstract}

\section{Introduction}

Cooking of meat is essential to achieve a palatable and safe product for consumer's acceptance. In general, cooking means the application of heat to meat following different procedures. The most common cooking techniques for meat are grilling, roasting, boiling, steaming and frying; all of them are carried out at different temperatures and length of cooking time.

There have been a considerable number of studies dealing with the physicochemical changes that underwent during meat cooking. ${ }^{[1][2]}$ Cooking loss is a combination of liquid and soluble matter lost during cooking. This is due to heat inducing protein denaturation during meat cooking, which causes less water to be entrapped within the protein structures. ${ }^{[3]}$ Moreover, King et al. found that heating caused myofibrillar shrinkage, decreasing sarcomere length which was linked to high cooking loss. ${ }^{[4]}$ The cooking loss depends on mass transfer during thermal treatment and therefore, different cooking methodologies will lead to different losses. ${ }^{[5]}$

Lipid oxidation is one of the adverse effects resulted from meat cooking and is reported as a major reason for meat deterioration and rancidity. Moreover, lipid oxidation products are implicated in several human pathologies such as atherosclerosis, cancer, inflammation and aging processes. ${ }^{[6]}$ Lipid oxidation is a complex process whereby hydroperoxides and other primary products of the oxidation are formed from polyunsaturated fatty acids. ${ }^{[7]}$ The primary autoxidation is followed by a series of secondary reactions which lead to the degradation of hydroperoxides and formation of a wide range compounds, including Malonaldehyde (MDA) or Thiobarbituric acid reactive substances (TBARs). This substance has become widely used as indicator of lipid oxidation.

There are many factors that influence lipid oxidation, including the composition and content of triglycerides, ${ }^{[8]}$ cooking conditions. ${ }^{[9]}$ For example, when different cooking methods were compared, roasting, which uses high temperatures for a long time, produces an increased lipid oxidation compared to other methods. ${ }^{[10]}$ Frying meat with oil can change the fatty acid composition of meat product and suffer oxidation. ${ }^{[6]}$ 
In Thailand, buffalo meat is produced from indigenous swamp buffalo and mostly used in traditional meat dish in house kitchen. However, to our knowledge, there is little information of the effect of cooking in buffalo meat quality. Therefore, this study was conducted to assess the influence of five different cooking methods (grilling, roasting, boiling, steaming and frying) on cooking loss and lipid oxidation of buffalo meat.

\section{Materials and methods}

Materials. The round bottom cuts (Semimembranosus) from 6 buffalo carcasses (of about 4-6 year's age) were collected after slaughter and kept under chilled condition for $24 \mathrm{~h}$. The cuts were then trimmed of separable fat and loose connective tissue.

Sample preparation. The cuts from each buffalo were sliced with meat slicer (SirMAN Suc V. Palladio, Italy) into $6 \mathrm{~mm}$ thick meat and divided into six groups according to the cooking methods. A total of 36 samples were obtained ( 6 muscles times 5 cooking methods plus one control group, raw meat), the treatment groups were cooked using the following methods: grilling at $125-135^{\circ} \mathrm{c}$ during $5 \mathrm{~min}$ on each surface, using an electrical griddle (OTTO, GR 141, China); roasting at $150{ }^{\circ} \mathrm{c}$ for 20 min using an electrical oven (Minoya, \#MOV $53 \mathrm{TM}$, Taiwan); boiling with water at $98{ }^{0} \mathrm{c}$ during $5 \mathrm{~min}$; steaming with water vapors at $98^{\circ} \mathrm{c}$ during $30 \mathrm{~min}$; frying using refined palm oil, at $175^{\circ} \mathrm{c}$ during 2 min on each surface. The cooking treatment was considered complete when all the samples had reached an internal temperature of $72^{\circ} \mathrm{c}$.

Cooking loss. After cooking, the samples were cooled at room temperature for $1 \mathrm{~h}$ and the percentage of cooking loss was recorded. The cooking loss was calculated as the percentage weight difference between fresh and cooked samples related to the weight of fresh sample.

TBARs measurement. The 2 thiobarbituric acid (TBARs) assay was carried out according to the extraction method described by Salih et al. ${ }^{[11]}$ Briefly, $5 \mathrm{~g}$ of sample were homogenized in polypropylene tubes of $50 \mathrm{ml}$ using a homogeneizer with $15 \mathrm{ml}$ of $3.86 \%$ perchloric acid and 0.50 $\mathrm{ml}$ of BHT (butylated hydroxytoluene) for $30 \mathrm{~s}$ and centrifuged at $3500 \mathrm{rpm}$ for $2 \mathrm{~min}$. During homogenization tubes were kept in ice to avoid heating. The blended sample was filtered through Whatman No.1 filter paper. $2 \mathrm{ml}$ aliquots of the filtrate were then mixed in glass tubes with $2 \mathrm{ml}$ of $0.02 \mathrm{M}$ TBA and shaked. The solution was heated at $100{ }^{\circ} \mathrm{c}$ for $30 \mathrm{~min}$, and then cooled under running water. The absorbance of solution was measured at $532 \mathrm{~nm}$ on a spectrophotometer against blank. Blank contains $2 \mathrm{ml}$ of perchloric acid (3.86\%) and $2 \mathrm{ml}$ of TBA reagent. The concentration of TBARs was calculated from a standard curve using 1,1,3,3- tetraethoxypropane (TEP). Results were expressed as mg malonaldehyde (MDA) per $\mathrm{kg}$ of meat sample.

Statistical analysis. The effect of different cooking treatments on cooking loss and TBARs index were examined on the same experimental unit (meat sliced from same round cut of each buffalo), therefore the data were subjected to analyze as a repeated measure. ${ }^{[12]}$ When a significant effect $(P<0.05)$ was detected, means were compared using Duncan's new multiple range test. Correlation between cooking loss and TBARs index was determined by correlation analysis using Pearson's linear correlation coefficient.

\section{Results and discussion}

The effect of different cooking methods on cooking loss of buffalo meat is shown in Fig 1. It can be seen that cooking losses were significantly $(P<0.05)$ affected by cooking treatments, being higher after steaming (49\%) and lower after grilling (39\%) and roasting (42\%), even though the two latter were cooked in the higher thermal temperature. Lorenzo et al. also reported the low cooking loss in foal samples cooked in the grill. ${ }^{[2]}$ They purposed the effect of grilling on low cooking loss that this could be due to the formation of defined crust on the meat surface which physically trapped water in the interior of the product. In addition, Dominguez et al. found the roasted foal steaks showed a decreased cooking loss compared to microwave treatment. ${ }^{[13]}$ They suggested the roasting created an external hard layer around the sample that slowed the exit of seeping liquid. With these regards, the presence of crust that occurred during cooking (grilling and roasting) could slowed the 
exit of seepage and prevent high cooking loss. On the contrary, the high cooking loss in steaming treatment was suggested that this was due to the fact that no crust formed during cooking.

The effect of different cooking methods on lipid oxidation expressed as mg MDA $/ \mathrm{kg}$ meat is presented in Fig.2. Values obtained were similar to those found by Kesava et al. in raw and cooked (broiled) buffalo meat. ${ }^{[14]}$ As it could be thought, cooking significantly increases MDA content in the products $(P<0.05)$. In this respect, there are many studies showing higher levels of MDA in different kinds of cooked meat in comparison to the raw meat. ${ }^{[15][16]}$

It seems that high temperature during cooking causes an increase of the oxidation processes in meat. In this study, grilled buffalo meat had the highest MDA level compared to lowest MDA concentration in boiled and steamed meat $(P<0.05)$. It is consistent with Juarez et al. who revealed grilling increased more TBARs content in buffalo meat than boiling. ${ }^{[3]}$ Thus, the application of higher thermal temperature $\left(125-135^{\circ} \mathrm{c}\right)$ in the grilling compared to the boiling and steaming with lower temperature $\left(98^{0} \mathrm{c}\right)$ could be the case. However, it should be noticed the roasting method, despite the high temperature $\left(150^{\circ} \mathrm{c}\right)$ used in the process, did not show statistic difference in MDA content compared to the boiling cooking method $(P>0.05)$. This indicated the different temperatures in cooking process did not solely affect the MDA level of the product.

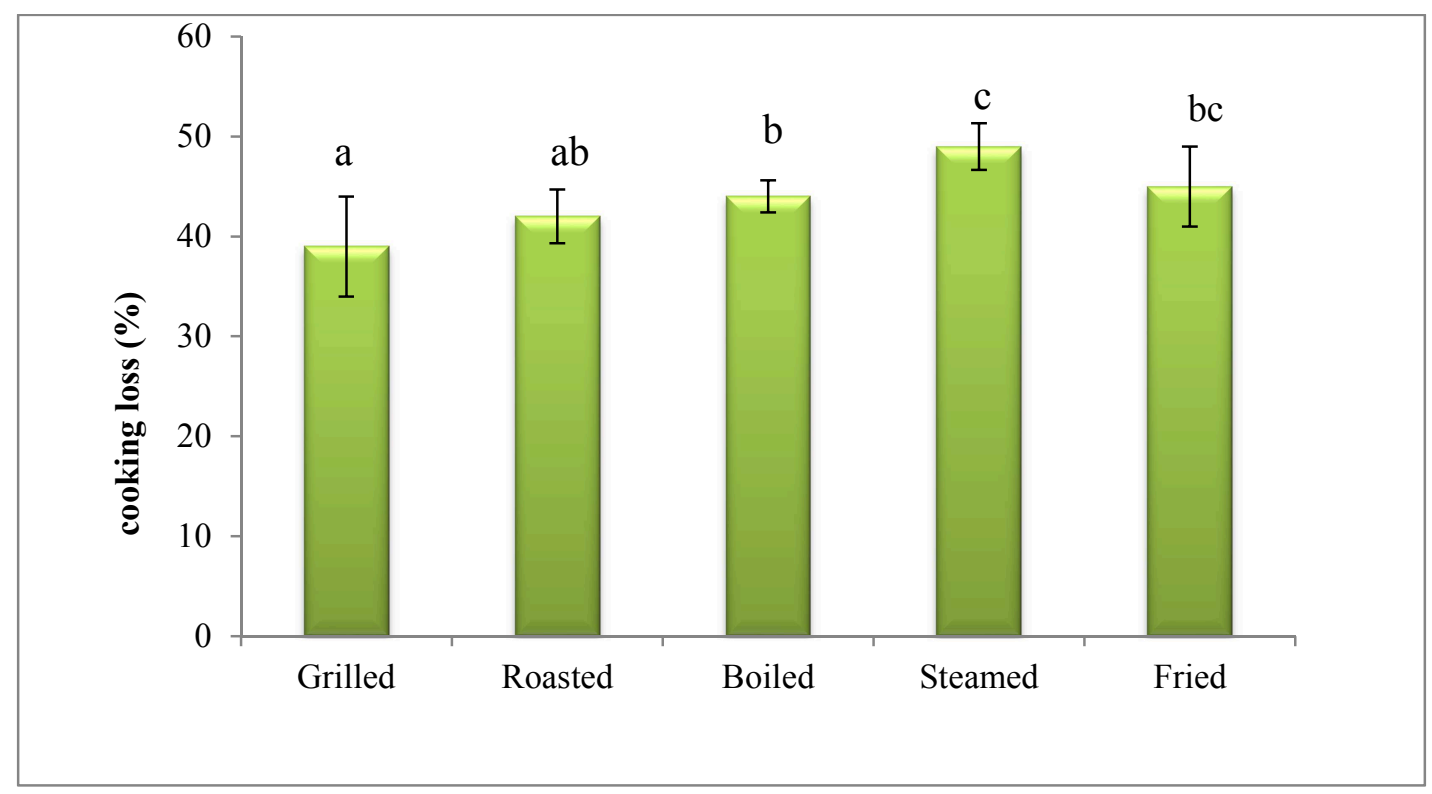

Fig.1 Effect of cooking methods on cooking loss of buffalo meats (mean \pm standard error).

${ }^{\mathrm{a}-\mathrm{c}}$ Different letters indicate significant differences at $P<0.05$.

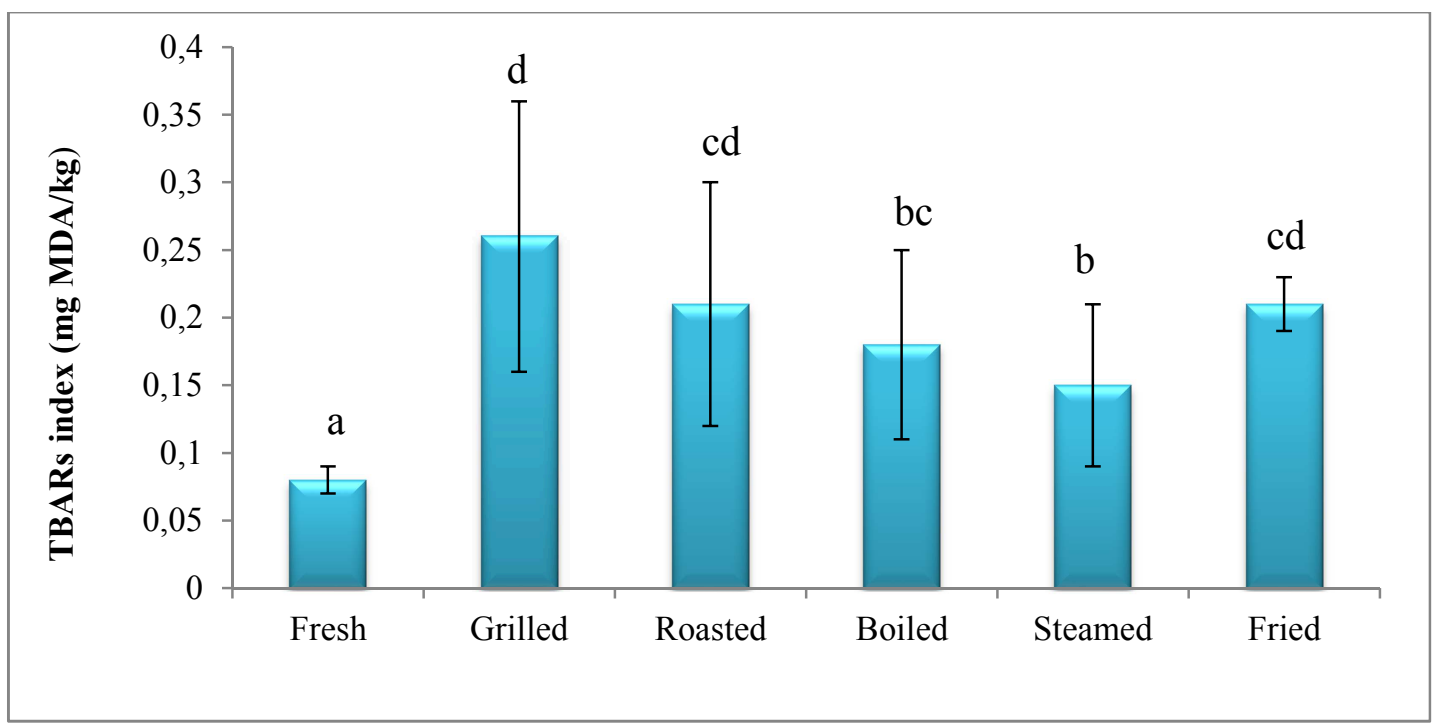

Fig. 2 Effect of cooking methods on TBARs values of buffalo meats (mean \pm standard error). ${ }^{\text {a-d }}$ Different letters indicate significant differences at $P<0.05$. 
In the current study, MDA concentration was related to the percentage of cooking loss. The Pearson s correlation coefficient between MDA concentration and percentage of cooking loss was found to be $-0.54(P<0.05)$. This was suggested that cooking loss could play role in the MDA level in the meat product. It could be possible due to the fact that MDA could dissolve with water. ${ }^{[17]}$ So, the MDA that generated during cooking could be loss from the product through seepage. If it is such a case, then, the cooking method that prevailed high cooking loss could be less in MDA level. This suggestion could be true in this study, since the higher cooking loss seen in the steaming had the lower level of MDA compared to the grilling which had lesser extent of cooking loss and prevailing the MDA content. On the other hand, the cooking procedures that had the similar degree of cooking loss did not show the difference in MDA level.

In conclusion, the different cooking methods resulted in difference in cooking loss and MDA level. Grilling with the lower cooking loss had the higher of MDA level compared to boiling and steaming cooking method. Cooking loss has shown negative correlation to MDA content and it was postulated that the cooking loss of meat could possibly be the response to the MDA level in the product.

\section{Acknowledgements}

We wish to acknowledge the Research and Development Institute, Kamphaeng Phet Rajabhat University for financing this research project.

\section{References}

[1] C.P.M. Alfaia, S.P. Alves, A.F. Lopes, M.F.E. Fernandes, A.S.H. Costa, C.M.G.A. Fontes, M.L.F. Castro, R.J.B. Bessa, J.A.M. Prates, Effect of cooking methods on fatty acids, conjugated isomers of linoleic acid and nutritional quality of beef intramuscular fat, Meat Sci. 84 (2010) 769777.

[2] J.M. Lorenzo, A. Cittadini, P.E. Munekata, R. Domínguez, Physicochemical properties of foal meat as affected by cooking methods, Meat Sci. 108 (2015) 50-54.

[3] M. Juárez, S. Failla, A. Ficco, F.Pena, C. Avilés, O. Polvillo, Buffalo meat composition as affected by different cooking methods, Food Bioproducts Process. 88 (2010) 145-148.

[4] D.A. King, M.E. Dikeman, T.L. Wheeler, C.L. Kastner, M. Koohmaraie, Chilling and cooking rate effects on some myofibrillar determinants of tenderness of beef, J. Anim. Sci. 81 (2003) 14731481.

[5] Q. Cheng, D.W. Sun, Factors affecting the water holding capacity of red meat products: A review of recent research advance, Critical Rev. Food Sci. Nutri. 48 (2008) 137-159.

[6] J.M. Broncano, M.J. Petrón, V. Parra, M.L. Timón, Effect of different cooking methods on lipid oxidation and formation of free cholesterol oxidation products (COPs) in latissimusdorsi muscle of Iberian pigs, Meat Sci. 83 (2009) 431-437.

[7] F. Shahidi, Y. Zhong, Lipid oxidation and improving the oxidative stability, Chem. Soc. Rev. 39 (2010) 4067-4079.

[8] A.I. Carrapiso, Effect of fat content on flavour release from sausages, Food Chem. 103 (2007) 396-403

[9] D.V. Byrnea, W.L.P. Brediea, D.S. Mottram, M. Marten, Sensory and chemical investigation on the effect of oven cooking on warmed-over flavor development in chicken meat, Meat Sci. 61 (2002) 127-139.

[10] P. Hernández, J.L. Navarro, F. Toldrá, Lipids of pork meat as affected by various cooking techniques, Food Sci.Tech.Interl. 5 (1999) 501-508. 
[11] A.M. Salih, D.M. Smith, J.F. Price, I.E. Dawson, Modified extraction 2- thiobarbituric acid method for measuring lipid oxidation in poultry, Poultry Sci. 66 (1987) 1483-1488.

[12] J.L. Myers, A.D. Well, Research Design and Statistical Analysis, Lawrence Erlbaum Associates, Inc.,Publishers, New Jersey, 1995.

[13] R. Domínguez, M. Gómez, S. Fonseca, J.M. Lorenzo, Influence of thermal treatment on formation of volatile compounds, cooking loss and lipid oxidation in foal meat, LWT- Food Sci. Tech. 58 (2014) 430-445.

[14] V. Kesava Rao, B.N. Kowale, N.P. Babu, G.S. Bisht, Effect of cooking and storage on lipid oxidation and development of cholesterol oxidation products in water buffalo meat, Meat Sci. 43 (1996) 179-185.

[15] A. Conchillo, D. Ansorena, I. Astiasarán, Combined effect of cooking (grilling and roasting) and chilling storage (with and without air) on lipid and cholesterol oxidation in chicken breast, J. Food Prot. 66 (2003) 840-846.

[16] M.N. O’Grady, R. Carpenter, P.B. Lynch, N.M. O’Brien, J.P. Kerry, Addition of grape seed extract and bearberry to porcine diets: Influence on quality attributes of raw and cooked pork, Meat Sci. 78 (2008) 438-446.

[17] V. Nair, C.L. O’Neil, P.G. Wang, Malondialdehyde, e- Eros encyclopedia of reagents for organic synthesis, 2008, http://dx.doi.org/doi:10.1002/047084289x.rm013.pub2. 